\title{
Hacia una integración de mindfulness e inteligencia emocional en psicología y educación
}

\author{
Towards the integration of mindfulness and emotional intelligence \\ in psychology and education
}

\author{
Manolete S. Moscoso ${ }^{\mathrm{a}, \mathrm{b}, *}$

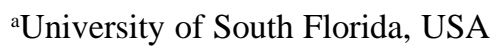 \\ bInstituto de Investigación en Psicología, Universidad de San Martin de Porres, Perú
}

\section{Resumen}

Antecedentes: el presente artículo presenta una revisión empírica de la integración de mindfulness e inteligencia emocional. Mindfulness es un modelo de intervención cognitiva que facilita la toma de consciencia con la experiencia en el momento presente, favoreciendo la autorregulación emocional. Igualmente, la inteligencia emocional ha sido conceptualizada como una constelación de habilidades relacionadas con la percepción, entendimiento y un adecuado manejo de las emociones propias y de los demás. Ambos marcos conceptuales están asociados con un buen sentido de bienestar y desarrollo personal. Estado del arte: se presenta una revisión de la evidencia científica preliminar publicada en la literatura psicológica y de educación, examinando los efectos mediadores de ambos constructos en el proceso de regulación emocional y calidad de vida individual. Conclusiones: los reportes de los estudios evaluados indican que la interacción de mindfulness disposicional e inteligencia emocional ofrece beneficios significativos en los estados de bienestar de las personas. De igual manera, se reportó que la inteligencia emocional es un mediador entre mindfulness e incrementos en las emociones positivas. El propósito del presente artículo de revisión es identificar un modelo integrador en la regulación de la emociones, incluyendo los «ingredientes activos» que influyen en los estados de bienestar y la forma en que la práctica de mindfulness contribuye al aumento de las habilidades de la inteligencia emocional.

Palabras clave: integración, mindfulness, inteligencia emocional, sentimiento de bienestar, distrés emocional.

\section{Para citar este artículo:}

Moscoso, M. S. (2019). Hacia una integración de mindfulness e inteligencia emocional en psicología y educación. Liberabit, 25(1), 107117. doi: https://doi.org/10.24265/liberabit.2019.v25n1.09

\begin{abstract}
Background: this article presents an empirical review of the integration of mindfulness and emotional intelligence. Mindfulness is a cognitive intervention model that helps people to develop awareness of their experience in the present moment favoring emotional self-regulation. Similarly, emotional intelligence has been conceptualized as a constellation of abilities related to the perception, understanding and adequate management of emotions within and among people. Both conceptual models are associated with a good sense of well-being and personal development. State of the art: this paper reviews the preliminary scientific evidence published in psychology and education literature, and examines the mediating effects of these two constructs in the process of emotional regulation and quality of life of individuals. Conclusions: the study results indicate that the interaction of mindfulness and emotional intelligence offers important benefits for people's wellbeing. It was also reported that emotional intelligence acts as mediator between mindfulness and greater positive emotions. The purpose of this review article is to identify a model of integration for emotional regulation that includes «active ingredients» to influence well-being and the way in which the practice of mindfulness contributes to increase the abilities of emotional intelligence.
\end{abstract}

Keywords: integration, mindfulness, emotional intelligence, well-being, emotional distress.

Este es un artículo Open Access bajo la licencia Creative Commons Atribución-NoComercial-CompartirIgual 4.0

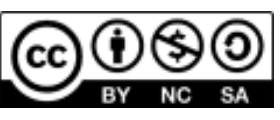




\section{Introducción}

Mayer y Salovey (1997) definen el concepto de inteligencia emocional como «la habilidad de percibir las emociones de manera precisa; la capacidad para comprenderlos de una manera óptima y adecuada; y la habilidad de regular dichas emociones con el propósito de promover el desarrollo personal» (p. 5). Con base a esta definición operacional, puede indicarse que la inteligencia emocional está representada por tres dimensiones claramente definidas: (1) la percepción de los estados emocionales, (2) un adecuado entendimiento de la naturaleza afectiva y (3) la capacidad de regulación emocional como una forma de desarrollo personal. La premisa lógica de este marco conceptual nos permite admitir que una persona emocionalmente inteligente presenta un conjunto de habilidades en función a una adecuada regulación emocional, las cuales contribuyen a mantener un nivel óptimo de adaptación en las relaciones interpersonales y en el manejo adecuado del estrés cotidiano (Slaski \& Cartwright, 2003).

Estudios correlacionales indican la importancia de la inteligencia emocional en el rendimiento académico, calidad de vida, salud y bienestar personal (Martins, Ramalho, \& Morin, 2010; Perera \& DiGiacomo, 2013; Sánchez-Álvarez, Extremera, \& FernándezBerrocal, 2016). Los datos reportados en estas investigaciones confirman la relevancia de este concepto en las áreas de la psicología de la salud y de la educación, y a la vez estimulan el interés por la aplicación de programas de intervención psicológica dirigidos a promover la inteligencia emocional (Campo, Laborde, \& Weckemann, 2015; Hodzic, Scharfen, Ripoll, Holling, \& Zenasni, 2017; Kidwell, Hasford, \& Hardesty, 2015). Este interés es relativamente nuevo y puede ser factible a través de modelos de intervención cognitiva basados en mindfulness (Schutte, Malouff, \& Thorsteinsson, 2013).

Mindfulness es descrita como la práctica de enfocar intencionalmente la atención en el momento presente sin elaborar juicios de valor (Kabat-Zinn, 1990). A la fecha, existen más de 3500 investigaciones publicadas utilizando los Programas de Reducción de Estrés basado en Mindfulness (Kabat-Zinn, 1990) y la Terapia Cognitiva basada en Mindfulness (Segal, Williams, \& Teasdale, 2002; Teasdale et al., 2000). Estos reportes indican que ambas formas de intervención generan efectos positivos en la regulación emocional (Chambers, Gullone, \& Allen, 2009; Hayes \& Feldman, 2004; Lengacher et al., 2007); existiendo consenso de que dichos modelos han sido desarrollados con la intención de reducir los niveles de distrés emocional, reducir la frecuencia de recurrencia de los estados depresivos y elevar los niveles de bienestar personal (Moscoso, Reheiser, \& Hann, 2004; Segal et al., 2002).

\section{Intergración de mindfulness e inteligencia emocional}

Desde una perspectiva integradora, el concepto de mindfulness es consistente y se superpone con el constructo de inteligencia emocional. El avance empírico de este modelo de intervención ha generado un terreno propicio para examinar científicamente la integración de la práctica de mindfulness y el constructo de inteligencia emocional como una variable mediadora en la reducción del distrés emocional y promover la regulación emocional. Esta visión representa una nueva línea de investigación con la noción de lograr un mejor entendimiento de los niveles de eficacia de mindfulness y su influencia sobre el posible incremento en las habilidades de la inteligencia emocional.

Los recientes estudios en esta área han sido estimulados por el desarrollo de dos campos de investigación importantes. Uno de ellos son los estudios de Fredrickson y colaboradores, los cuales han permitido observar un interés creciente por el estudio de la práctica de mindfulness y el rol que las emociones positivas cumplen dentro del proceso de regulación emocional (Garland, Farb, Goldin, \& Fredrickson, 2015; Tugade \& Fredrickson, 2004). Estos estudios señalan que el incremento de las emociones positivas amplían el control atencional y de acción del individuo, facilitando de esta manera 
un conjunto de recursos favorables para el desarrollo de atributos personales y de resiliencia hacia la adversidad (Garland, Gaylord, \& Fredrickson 2011).

El otro se refiere a las recientes investigaciones correlacionales en relación a mindfulness e inteligencia emocional. Estas han sido realizadas con la noción de examinar las asociaciones entre ambos constructos dentro de la psicología de la salud y bienestar personal (Bao, Xue, \& Kong, 2015; Kidwell et al., 2015; Ramos \& Hernández, 2008; Schutte \& Malouff, 2011; Teal et al., 2018; Wang \& Kong, 2014). Dichos estudios reportan que ambos modelos presentan implicancias de carácter mediador con los estados de bienestar personal, calidad de vida, distrés emocional y estrés percibido. Igualmente, se han reportado resultados preliminares en función a la utilización de estrategias cognitivas sobre la regulación emocional en estudiantes universitarios (Ramos \& Hernández, 2008).

La hipótesis general de esta integración sugiere que los niveles elevados de mindfulness disposicional están significativamente correlacionados con un adecuado nivel de regulación emocional (Baer et al., 2008; Brown \& Ryan, 2003; Kidwell et al., 2015). En congruencia con la premisa de mindfulness disposicional, se asume que un nivel adecuado de inteligencia emocional está asociada positivamente con un ajuste óptimo de regulación emocional (Mayer \& Salovey, 1997). Esta asociación implícita y la mediación de la inteligencia emocional en la práctica de mindfulness tienen implicancias muy importantes en la calidad de vida individual (Lengacher et al., 2009; Schutte \& Malouff, 2011; Teal et al., 2018).

El tema central del presente artículo está en función a las siguientes interrogantes: ¿Es posible integrar los paradigmas de mindfulness e inteligencia emocional? Los estudios iniciales publicados en esta línea de investigación ofrecen evidencias de que dicha integración y relación mediadora es posible. Si esta asociación entre ambos modelos es factible, ¿cómo y de qué manera la práctica de mindfulness posibilita los efectos positivos en la adquisición de habilidades que caracterizan a la inteligencia emocional? Con base a estos dos planteamientos, el propósito del presente estudio es realizar una revisión de la literatura pertinente acerca de las asociaciones mediadoras de mindfulness e inteligencia emocional, así como también, sugerir un marco integrador y los posibles «ingredientes activos» de mindfulness que estimulan la inteligencia emocional y sus efectos mediadores sobre los estados de bienestar personal y reducción del estrés percibido (Bao et al., 2015; Schutte \& Malouff, 2011).

\section{Elementos integradores de mindfulness e inteligencia emocional}

Las definiciones conceptuales de mindfulness presentada por Kabat-Zinn (1990) y de inteligencia emocional expuesta por Mayer y Salovey (1997) están descritas claramente en términos de los procesos regulatorios de las emociones. Este planteamiento integrador toma en consideración el rol central que cumple la regulación emocional en la aplicación práctica de ambos modelos, asumiendo implícitamente que la inteligencia emocional y el rasgo mindfulness disposicional coexisten mutuamente y están interconectados en una dirección correlacional positiva. Por lo tanto, la asociación entre ambos constructos debe ser entendida en función al incremento de emociones positivas y bienestar personal, y/o la disminución de emociones negativas y del distrés emocional.

La definición de mindfulness toma en consideración dos elementos básicos en el proceso de regulación emocional: (a) la autorregulación de la atención en el momento presente y (b) la minimización de los juicios de valor (Bishop et al., 2004; Moscoso \& Lengacher, 2015). Kabat-Zinn (1990) refiere que cuando una persona observa la experiencia del momento presente bajo el lente de mindfulness, invariablemente comienza a apreciar dichos eventos desde una perspectiva nueva debido a los cambios en la percepción de dicho individuo. Por lo tanto, el entrenamiento de mindfulness genera sus efectos a través de la regulación emocional y dicho «cambio 
de percepción». Por otro lado, la noción de inteligencia emocional presenta una clara distinción de sus dos componentes principales: (a) la inteligencia como una habilidad de carácter cognitiva y (b) la emoción como un estado afectivo con la capacidad inherente de modificar el pensamiento en función a la regulación emocional (Salovey \& Mayer, 1990).

Con base a este modus operandi del marco conceptual de ambos constructos, el proceso de regulación emocional constituye el primer elemento integrador en dicha asociación por cuanto representa una forma estratégica de lograr las metas propuestas en el marco de la práctica de mindfulness, como también en la adquisición de las habilidades de la inteligencia emocional. Desde la perspectiva de mindfulness, este proceso de regulación emocional esta esencialmente influenciada por el «sostenimiento de la atención» en los pensamientos y emociones de la experiencia presente. Desde el punto de vista de la inteligencia emocional, dicho proceso de regulación emocional está en función a la «habilidad de percibir» las emociones propias con una orientación adaptativa.

Un segundo mecanismo de integración está en función a la adquisición de habilidades cognitivoemocionales inherentes en ambos modelos conceptuales, tales como la sostenibilidad de la atención y toma de conciencia en la práctica de mindfulness; así como la habilidad de percibir y entender las emociones desde la perspectiva de la inteligencia emocional. En el caso de mindfulness, la adquisición de estas habilidades a través de la práctica y entrenamiento formal tiene implicancias significativas en el incremento de emociones positivas y fomentan un ajuste adaptativo a las situaciones de estrés (Garland et al., 2011). En lo concerniente a la inteligencia emocional, es el aprendizaje de las habilidades y atributos que la caracterizan.

Un tercer elemento integrador de mindfulness e inteligencia emocional está sustentado por los estudios científicos realizados dentro de la psicología positiva en la última década. Esta corriente psicológica está orientada al estudio de las emociones positivas y sus efectos sobre la salud, bienestar y desarrollo personal del individuo. En este contexto, las emociones positivas emergen como un factor vital en la adquisición de características resilientes en el individuo (Garland et al., 2011). Por ejemplo, el estudio llevado a cabo por Tugade y Fredrickson (2002) sugiere que individuos con características resilientes tienen la habilidad de percibir con mayor claridad los beneficios producidos por la experiencia de emociones positivas, todas ellas metas razonables de mindfulness y de la inteligencia emocional.

\section{Evidencia empírica de integración}

El interés por examinar la asociación entre inteligencia emocional y la práctica de mindfulness se encuentra actualmente en vías de desarrollo. Actualmente, se están llevado a cabo estudios empíricos de carácter exploratorio con el propósito de evaluar las interacciones entre ambos constructos. Con base a los resultados preliminares, se puede indicar que los datos reportados muestran una estrecha relación mediadora con variables relacionadas a la regulación emocional y salud mental; y de manera particular, la reducción del distrés emocional, ansiedad y mejoramiento en el bienestar personal de las personas (Bao et al., 2015; Ramos, Hernández, \& Blanca, 2009; Schutte \& Malouff, 2011; Teal et al., 2018; Wang \& Kong, 2014).

El primer estudio controlado fue llevado a cabo por Ramos et al. (2009) con el propósito de examinar los niveles de eficacia de ambos modelos integrados en la regulación emocional utilizando una muestra de 49 estudiantes universitarios. Los autores reportaron una reducción de la ansiedad en el grupo experimental y cambios significativos en la utilización de las estrategias de reducción emocional e incrementos del afecto positivo; concluyendo que la integración de ambos modelos «genera un beneficio emocional a corto plazo» en dicha muestra (p. 207). 
Schutte y Malouff (2011) realizaron un estudio correlacional con la intención de evaluar el rol mediador de la inteligencia emocional en la práctica de mindfulness y sus efectos sobre el bienestar personal en una muestra de 125 estudiantes universitarios. Los resultados observados indican que niveles elevados de mindfulness están significativamente correlacionados con niveles elevados de inteligencia emocional, afecto positivo y satisfacción personal; y con una reducción en el afecto negativo. Los autores concluyen que «los resultados del estudio ofrecen evidencias de una asociación entre ambos constructos y el bienestar personal percibido» (p. 1118).

Otra investigación realizada con 321 adultos examinó el rol de la inteligencia emocional en función al impacto de mindfulness sobre las variables de distrés emocional y satisfacción personal (Wang \& Kong, 2014). Los resultados corroboran el rol de la inteligencia emocional en la práctica de mindfulness y cómo este modelo de intervención produce sus efectos positivos sobre la salud de las personas. Los autores indican que «los resultados presentan evidencias significativas de validez externa de mindfulness como predictor del distrés emocional y satisfacción personal en dicha muestra» (p. 850).

Bao y colaboradores diseñaron un estudio correlacional con una muestra de 380 adultos, en el cual se examinó las relaciones entre inteligencia emocional, mindfulness y estrés percibido (Bao et al., 2015). Los resultados reportan que «mindfulness está correlacionada positivamente con inteligencia emocional, y negativamente asociada con el estrés percibido; es decir, las personas con elevados niveles de mindfulness tienden a hacer uso de sus emociones con el propósito de motivarse a sí mismos y mejorar sus comportamientos, reduciendo de esa manera sus niveles de estrés percibido» (Bao et al., 2015, p. 51).

Teal y asociados reportaron los resultados de un estudio realizado con un grupo de 294 jóvenes adolescentes (edades: 13 a 17 años), en el cual examinaron la relación entre inteligencia emocional, mindfulness disposicional y bienestar personal (Teal et al., 2018). Los autores concluyen que la habilidad de una persona para manejar las emociones positivas y negativas podría estar interconectada con la atención y conciencia de sus pensamientos estimulando un buen estado de bienestar psicológico.

En adición a estos resultados, los estudios aleatorizados en pacientes con cáncer llevados a cabo en nuestro laboratorio presentan evidencias de un mejoramiento significativo en la calidad de vida y reducción de distrés emocional, lo cual permite sugerir que la práctica de mindfulness genera un estado de conciencia metacognitiva y favorece el proceso de regulación emocional y reducción del distrés emocional, ampliando la postura perceptiva de la persona y posibilitando una adaptación adecuada frente a las experiencias de adversidad y estrés relacionados con el diagnóstico de cáncer (Lengacher et al., 2014; Moscoso, 2018; Moscoso \& Lengacher, 2015).

\section{Mediación cognitiva y mecanismos de acción}

Los hallazgos de los estudios preliminares reportados en párrafos anteriores acerca de las relaciones entre mindfulness e inteligencia emocional inducen a plantear premisas que explican los mecanismos de acción del entrenamiento de mindfulness como posibles efectos sobre la inteligencia emocional. Una revisión del estudio de Teal et al. (2018) indica que la habilidad de lograr un nivel de autorregulación de las emociones positivas y negativas están asociadas con la atención y toma de conciencia de estas cualidades afectivas, generando de esta manera un estado de bienestar personal. Igualmente, el trabajo de Bao et al. (2015) reporta que los individuos con niveles elevados de mindfulness disposicional tienden a manejar sus emociones de una forma proactiva con la intención de reducir los niveles de distrés emocional. La investigación de Schutte y Malouff (2011) refiere que niveles altos de mindfulness están positivamente correlacionados con grados elevados de inteligencia emocional y satisfacción personal. 
Los mecanismos de acción de mindfulness han sido descritos con anterioridad (Moscoso \& Lengacher, 2015; Moscoso \& Lengacher, 2017; Moscoso, 2018). En ciertos casos, los modos de acción son específicos a los síntomas sobre los cuales la práctica de mindfulness ejerce sus efectos positivos. En función a la inteligencia emocional, estos ingredientes activos presentan una variación relativa tratándose de un atributo cognitivo-emocional, en lugar de un síntoma psicológico. Los estudios empíricos revisados en este artículo sugieren que el proceso de regulación emocional es el pilar de la integración entre ambos modelos conceptuales. En este sentido, es importante plantear tres mecanismos de acción específicos por los cuales la práctica de mindfulness estimula la inteligencia emocional con la intención de reducir los niveles de distrés emocional y elevar el bienestar personal: (a) el control y sostenibilidad de la atención, (b) el distanciamiento cognitivo o decentering y (c) la revaloración cognitiva de las emociones o mindful reappraisal.

\section{La controlabilidad y sostenimiento de la atención}

La inteligencia emocional es un atributo aprendido, el cual se caracteriza por la habilidad de percibir las emociones de manera precisa y adecuada. Esta conceptualización parcial de inteligencia emocional permite sugerir un primer mecanismo de acción: la controlabilidad y sostenimiento de la atención. La práctica de mindfulness enfatiza el enfoque de la atención en la respiración, permitiendo tomar conciencia de las «distracciones» de la mente que ocurren de manera habitual. La función del participante de mindfulness es reconocer la presencia de emociones positivas y negativas en la experiencia del momento presente y con una postura de ecuanimidad refocalizar la atención en la respiración. Esta forma de entrenamiento facilita la sostenibilidad de la atención focalizada con la intención de incrementar la observación en la calidad afectiva experimentada (Jha, Krompinger \& Baime, 2007; Moscoso, 2018).

La controlabilidad y sostenimiento de la atención de los estados afectivos es claramente un modo de acción en la mediación de la inteligencia emocional sobre el bienestar personal del participante (Teal et al., 2018). Este proceso cognitivo, mejor conocido como atención ejecutiva o percepción de conflictos emocionales, es de interés en neurociencia cognitiva (Tang et al., 2007). Dicho mecanismo de acción es un elemento fundamental en la práctica de mindfulness y cumple un rol central en la «percepción» de las emociones de una manera precisa y adecuada como postula el modelo de inteligencia emocional. Evidencia científica basada en imágenes de resonancia magnética funcional (fMRI), demuestra que la sostenibilidad de la atención ejecutiva durante la práctica de mindfulness genera incrementos en la actividad de la corteza cingulada anterior (Lane et al., 1998). En consistencia con los estudios de Lane, las investigaciones de Hölzel indican que participantes con experiencia en la práctica de mindfulness presentan niveles elevados de activación de la corteza cingulada anterior (Hölzel et al., 2008).

\section{Distanciamiento cognitivo o decentering}

El segundo modo de acción de mindfulness tiene relación con la postura de tomar distancia cognitivamente (decentering). Este mecanismo se expresa durante la fase del «monitoreo abierto» descrita en el idioma inglés como open monitoring (Brown \& Ryan, 2003), facilitando una secuencia de pensamientos con la intención de observar y reconocer ciertos patrones emocionales y de conducta poniendo énfasis a «no caer en el juego rumiativo de las emociones negativas» (Fresco et al., 2007). Dentro del modelo de integración de mindfulness e inteligencia emocional, el mecanismo de decentering ofrece la oportunidad de estimular la percepción de los estados emocionales y regularlos adaptativamente como lo indica el marco conceptual de este último constructo. En este sentido, la regulación emocional, definida como la capacidad de controlar y manejar adecuadamente los impulsos y sentimientos en conflicto, está directamente relacionada con este modo de acción (Brackett, Rivers, \& Salovey, 2011; Moscoso \& Lengacher, 2017; Segal et al., 2002). 


\section{La revaloración cognitiva de las emociones o mindful reappraisal}

La revaloración cognitiva o mindful reappraisal es un estado metacognitivo, el cual transforma la postura observadora de las experiencias emocionales del momento presente y promueve un nuevo estado afectivo de carácter positivo (Garland et al., 2015). Este proceso representa el tercer ingrediente activo en la integración de mindfulness e inteligencia emocional. En la medida que la práctica de mindfulness progresa, el participante logra experimentar dicha transformación, generando un cambio de percepción en cuanto a las experiencias emocionales negativas y opta por una postura comportamental más adaptada a las circunstancias del momento presente, por ejemplo, una actitud de aceptación y en otros casos una postura de autocompasión. Este mecanismo de revaloración cognitiva observado en la práctica de mindfulness cumple un rol central en la adquisición de las habilidades que caracterizan el concepto de inteligencia emocional como mediador del bienestar personal (Schutte \&
Malouff, 2011; Teal et al, 2018) y de reducción del distrés emocional (Wang \& Kong, 2014).

La evidencia empírica reportada por Garland y colaboradores (2015) en relación al afecto positivo como resultado del mecanismo de acción megacognitivo de regulación emocional durante la práctica de mindfulness, ha sido también observada en nuestros estudios en pacientes con cáncer. Por ejemplo, al inicio del programa de mindfulness, las participantes «perciben» el diagnóstico de cáncer de mama como un posible riesgo a sus vidas; en la medida que el entrenamiento transcurre, las participantes adquieren una postura perceptiva más optimista en relación a sus temores y hábitos de rumiación causado por la ansiedad. La revaloración cognitiva o mindful appraisal transforma los temores iniciales en una postura positiva como percibir dicho diagnóstico como un «nuevo reto a sus vidas» (Lengacher et al., 2012; Lengacher et al., 2014).

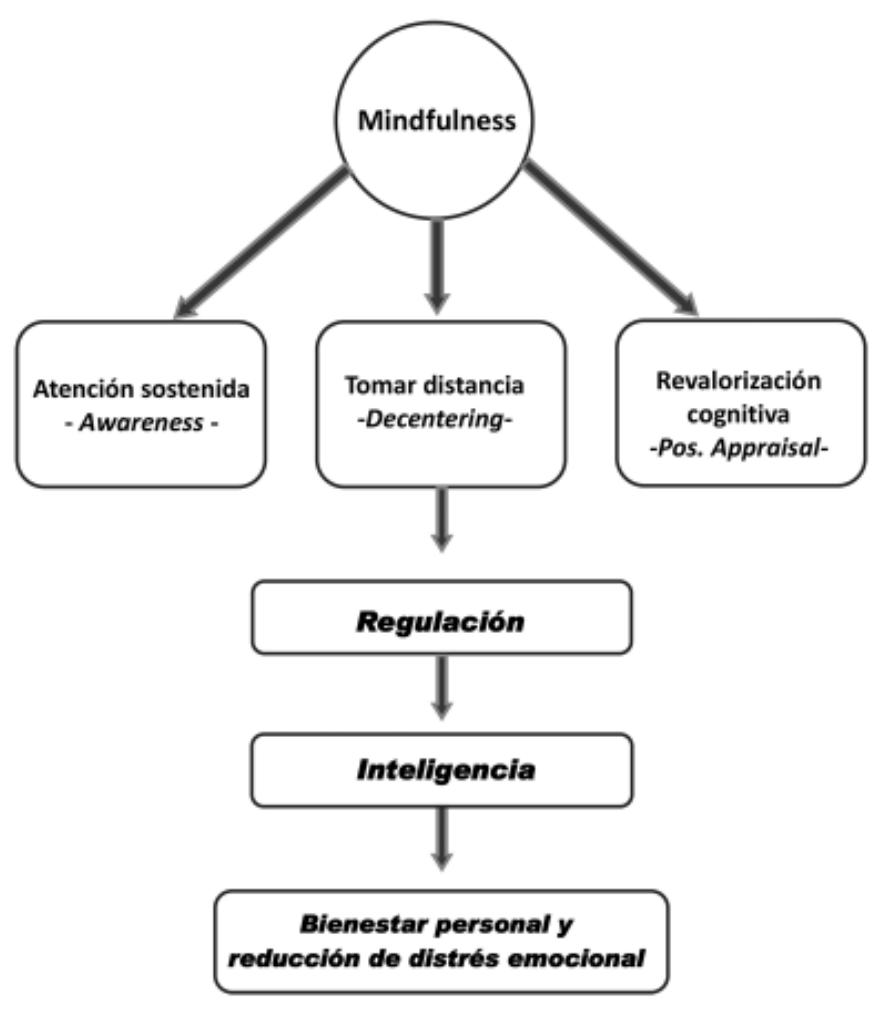

Figura 1. Integración y mediación de mindfulness e inteligencia emocional. 


\section{Discusión}

La inteligencia emocional es un constructo teórico de gran importancia en el campo de la psicología de la salud y educación por cuanto su utilidad está orientada al desarrollo personal del individuo, la habilidad de tomar decisiones y crear un estado de bienestar personal y, por último, la reducción de los niveles de distrés emocional en las relaciones interpersonales. La noción de asociar a las emociones al concepto tradicional de inteligencia en psicología fue considerado un modelo novedoso (Salovey \& Mayer, 1990), lo cual ha permitido que el estudio de esta área, en la actualidad, sea una de las más fértiles.

En el presente artículo, se considera a la inteligencia emocional como un modelo conceptual de habilidades posibles de ser adquiridas y mejoradas a través del entrenamiento de carácter cognitivo-emocional. Los reportes iniciales de Salovey y Mayer (1990) y de Mayer y Salovey (1997) presentan a este modelo como una forma de interacción entre la cognición y las emociones, indicando que estas últimas cumplen un rol central en el comportamiento adaptativo. El marco conceptual de habilidades que representan la inteligencia emocional están enmarcadas por tres componentes vitales relacionados con: (1) la percepción de los estados emocionales, (2) la comprensión de la naturaleza afectiva y (3) la habilidad de manejar el proceso de regulación emocional con el propósito de lograr un estado de bienestar y desarrollo personal.

Tomando en cuenta dicho contexto conceptual y la revisión de la literatura sobre las relaciones y mediación de la práctica de mindfulness sobre la inteligencia emocional, se sugiere la existencia de evidencia preliminar de que la constelación de habilidades que representan este constructo pueden ser estimuladas a través del entrenamiento de mindfulness, particularmente un aumento en el bienestar personal y calidad de vida, así como también una mejor adaptación en las relaciones interpersonales. La interacción de los mecanismos de acción inherentes a la práctica de mindfulness y las habilidades que caracterizan la inteligencia emocional facilitan una integración adecuada de ambos modelos conceptuales, tomando en consideración el rol central que cumple la regulación emocional dentro de este proceso integrativo.

La integración entre la práctica de mindfulness y el constructo de inteligencia emocional descrito en el presente artículo, ofrece un significativo potencial teórico y empírico. A pesar de ello, la actual evidencia empírica y mediaciones observadas deben ser consideradas de carácter preliminar. La dirección a seguir en el futuro requiere la evaluación de una mayor cantidad de estudios empíricos que permitan consolidar los resultados observados a la fecha. Los futuros estudios que se interesen por examinar la relación mediadora entre ambos modelos, deberán seguir incidiendo en el análisis de los efectos sobre el bienestar personal y reducción del distrés emocional en las áreas de la salud, educación y psicología.

\section{Conflicto de intereses}

El autor declara no tener ningún conflicto de intereses.

\section{Responsabilidad ética}

El autor declara que por tratarse de un artículo de revisión, los aspectos de protección de personas y animales, confidencialidad de datos y consentimiento informado no aplican al presente estudio. 


\section{Referencias}

Baer, R. A., Smith, G. T., Lykins, E., Button, D., Krietemeyer, J., Sauer, S., ... Williams, J. M. (2008). Construct validity of the five facet mindfulness questionnaire in meditating and nonmeditating samples. Assessment, 15(3), 329-342. doi: 10.1177/ 1073191107313003

Bao, X., Xue, S., \& Kong, F. (2015). Dispositional mindfulness and perceived stress: The role of emotional intelligence. Personality and Individual Differences, 78, 48-52. doi: 10.1016/j.paid.20 15.01.007

Bishop, S. R., Lau, M., Shapiro, S., Carlson, L., Anderson, N. D., Carmody, J., ... Devins, G. (2004). Mindfulness: a proposed operational definition. Clinical Psychology: Science and Practice, 11(3), 230-241. doi: 10.1093/clipsy.bph077

Brackett, M. A., Rivers, S. E., \& Salovey, P. (2011). Emotional Intelligence: Implications for personal, social, academic, and workplace success. Social and Personality Psychology Compass, 5(1), 88-103. doi: 10.1111/j.1751-9004.2010.00334.x

Brown, K. W., \& Ryan, R. M. (2003). The benefits of being present: Mindfulness and its role in psychological well-being. Journal of Personality and Social Psychology, 84(4), 822-848. doi: 10.10 37/0022-3514.84.4.822

Campo, M., Laborde, S., \& Weckemann, S. (2015). Emotional intelligence training: Implications for performance and health. In A. M. Colombus (Ed.), Advances in psychology research (pp. 75-92). New York: Nova.

Chambers, R., Gullone, E., \& Allen, N. B. (2009). Mindful emotion regulation: An integrative review. Clinical Psychology Review, 29, 560-572. doi: 10.10 16/j.cpr.2009.06.005

Fresco, D., Moore, M., Dulmen M. van, Segal, Z., Ma, S., Teasdale, J., \& Williams, J. (2007). Initial psychometric properties of the experiences questionnaire: validation of a self-report measure of decentering. Behavior Therapy, 38(3), 234-246. doi: 10.1016/j.beth.2006.08.003
Garland, E. L., Farb, N. A., Goldin, P., Fredrickson, B. L. (2015). Mindfulness broadens awareness and builds eudaimonic meaning: A process model of mindful positive emotion regulation. Psychological Inquiry, 26(4), 293-314. doi: 10.1080/1047840X.2015.1064 294

Garland, E. L., Gaylord, S. A., \& Fredrickson, B. L. (2011). Positive reappraisal mediates the stressreductive effects of mindfulness: An upward spiral process. Mindfulness, 2(1), 59-67. doi: 10.1007/s12 671-011-0043-8

Hayes, A. M., \& Feldman, G. (2004). Clarifying the construct of mindfulness in the context of emotion regulation and the process of change in therapy. Clinical Psychology: Science and Practice, 11(3), 255-262. doi: 10.1093/clipsy/bph080

Hodzic, S., Scharfen, J., Ripoll, P., Holling, H., \& Zenasni, F. (2018). How efficient are emotional intelligence trainings: A meta-analysis. Emotion Review, 10(2), 138-148. doi: 10.1177/1754073917708613

Hölzel, B. K., Ott, U., Hempel, H., Weygandt, M., Morgen, K., \& Vaitl, D. (2008). Investigation of mindfulness meditation practitioners with voxel-based morphometry. Social Cognitive and Affective Neuroscience, 3(1), 55-61. doi: 10.1093/scan/nsm038

Jha, A. P., Krompinger, J., \& Baime, M. J. (2007). Mindfulness training modifies subsystems of attention. Cognitive, Affective, \& Behavioral Neuroscience, 7(2), 109-119. doi: 10.3758/cabn.7.2.109

Kabat-Zinn, J. (1990). Full catastrophe living: Using the wisdom of your body and mind to face stress, pain, and illness. New York: Dell Publishing.

Kidwell, B., Hasford, J., \& Hardesty, D. M. (2015). Emotional ability training and mindful eating. Journal of Marketing Research, 52(1), 105-119. doi: 10.1509/ jmr.13.0188

Lane, R. D., Reiman, E. M., Axelrod, B., Yun, L. S., Holmes, A., \& Schwartz, G. E. (1998). Neural correlates of levels of emotional awareness: evidence of an interaction between emotion and attention in the anterior cingulated cortex. Journal of Cognitive Neuroscience, 10(4), 525-535. doi: 10.1162/089892 998562924 
Lengacher, C., Johnson-Mallard, V., Post-White, J., Moscoso, M., Jacobsen, P., Klein, T., ... Kip, K. (2009). Randomized controlled trial of mindfulnessbased stress reduction (MBSR) for survivors of breast cancer. Psycho-Oncology, 18(12), 1261-1272. doi: 10.1002/pon.1529: 10.1002/pon.1529

Lengacher, C. A., Kip, K. E., Moscoso, M., JohnsonMallard, V., Molinari, M., Gaurkee, D., ... Greenberg, H. (2007). Mindfulness-Based Stress Reduction (MBSR) improves psychological status and general health among breast cancer survivors. PsychoOncology, 16, 3, S72-S73.

Lengacher, C. A., Shelton, M. M., Reich, R. R., Barta, M. K., Johnson-Mallard, V., Moscoso, M. S., ... Kip, K. E. (2014). Mindfulness based stress reduction (MBSR (BC)) in breast cancer: evaluating fear of recurrence (FOR) as a mediator of psychological and physical symptoms in a randomized control trial (RTC). Journal of Behavioral Medicine, 37(2), 185195. doi: 10.1007/s10865-012-9473-6

Lengacher, C., Reich, R., Post-White, J., Moscoso, M., Shelton, M., Barta, M., ... Budhrani (2012). Mindfulness based stress reduction in post-treatment breast cancer patients: an examination of symptoms and symptom clusters. Journal of Behavioral Medicine, 35(1), 86-94. doi: 10.1007/s10865-011-9346-4

Martins, A., Ramalho, N., \& Morin, E. (2010). A comprehensive meta-analysis of the relationship beween emotional intelligence and health. Personality and Indivdual Differences, 49(6), 554564. doi: 10.1016/j.paid.2010.05.029

Mayer, J. D., \& Salovey, P. (1997). What is emotional intelligence? In P. Salovey \& D. J. Sluyter (Eds.), Emotional Development and Emotional Intelligence: Educational Implications, (pp. 3-34). New York: Harper Collins.

Moscoso, M. S. (2018). Mindfulness, autorregulación emocional y reducción de estrés: ¿ciencia o ficción? Revista Mexicana de Investigacion en Psicología, 10 (2), 101-121.

Moscoso, M. S., \& Lengacher, C. A. (2015). Mecanismos neurocognitivos de la terapia basada en mindfulness. Liberabit, 21(2), 221-233.
Moscoso, M. S., \& Lengacher, C. A. (2017). El rol de mindfulness en la regulación emocional de la depresión. Mindfulness \& Compassion. 2, 64-70. doi: 10.1016/j.mincom.2017.08.002

Moscoso, M. S., Reheiser, E. C., \& Hann, D. (2004). Effects of a brief mindfulness-based stress reduction intervention on cancer patients. Psycho-Oncology, 13(1), S12.

Perera, H. N., \& DiGiacomo, M. (2013). The relationship of trait emotional intelligence with academic performance: A meta-analytic review. Learning and Individual Differences, 28, 30-33. doi: 10.1016/j.lin dif.2013.08.002

Ramos, N. S., \& Hernández, S. M. (2008). Inteligencia emocional y mindfulness; hacia un concepto integrado de la inteligencia emocional. Revista Facultad de Trabajo Social, 24(24), 135-146. Recuperado de https://revistas.upb.edu.co/index.php/ trabajosocial/article/view/251

Ramos, N. S., Hernández, S. M., \& Blanca, M. J. (2009). Efecto de un programa integrado de mindfulness e inteligencia emocional sobre las estrategias cognitivas de regulación emocional. Ansiedad y Estrés, 15(2-3), 207-216. Recuperado de https://dialnet.unirioja.es/ servlet/articulo?codigo $=3095135$

Salovey, P., \& Mayer, J. D. (1990). Emotional intelligence, Imagination, Cognition, and Personality, 9(3), 185211. doi: 10.2190/DUGG-P24E-52WK-6CDG

Sánchez-Álvarez, N., Extremera, N. \& Fernández-Berrocal, P. (2016). The relation between emotional intelligence and subjective well-being: A meta-analytic investigation. The Journal of Positive Psychology, 11(3), 276-285. doi: 10.1080/17439760.2015.1058968

Schutte, N. S., \& Malouff, J. M. (2011). Emotional intelligence mediates the relationship between mindfulness and subjective well-being. Personality and Individual Differences, 50(7), 1116-1119. doi: 10.1016/J.paid.2011.01.037

Schutte, N. S., Malouff, J. M., \& Thorsteinsson, E. B. (2013). Increasing Emotional Intelligence through Training: Current Status and Future Directions. The International Journal of Emotional Education, 5(1), 56-72. Recuperado de https://core.ac.uk/download/ pdf/26812358.pdf 
Segal, Z. V., Williams, M. G., \& Teasdale, J. D. (2002). Mindfulness-Based Cognitive Therapy for Depression: A New Approach to Preventing Relapse. New York: Guilford Press.

Slaski, M., \& Cartwright, S. (2003). Emotional intelligence training and its implications for stress, health, and performance. Stress and Health Journal of the International Society for the Investigation of Stress, 19(4), 233-239. doi: 10.1002/smi.979

Tang, Y. Y., Ma, Y., Wang, J., Fan, Y., Feng, S., Lu, Q., ... Posner, M. I. (2007) Short-term meditation training improves attention and self-regulation. Proceedings of the National Academy of Sciences of the United States of America, 104(43), 17152-17156. doi: 10.1073/ pnas.0707678104

Teal, C., Downey, L. A., Lomas, J. E., Ford, T. C., Bunnett, E. ,R. \& Stough, C. (2018). The Role of Dispositional Mindfulness and Emotional Intelligence in Adolescent Males. Mindfulness, 10(1), 159-167. doi: 10.1007/ s12671-018-0962-8
Teasdale, J. D., Segal, Z. V., Williams, J. M. G., Ridgeway, V., Soulsby, J., \& Lau, M. (2000). Prevention of relapse/ recurrence in major depression by mindfulness-based cognitive therapy. Journal of Consulting and Clinical Psychology, 68(4), 615-623.

Tugade, M., \& Fredrickson, B. L. (2002). Positive emotions and emotional intelligence. In L. feldman Barret \& P. Salovey (Eds.). The wisdom of feelings (pp. 319-340). New York: Guilford.

Tugade, M., \& Fredrickson, B. L. (2004). Resilient individuals use positive emotions to bounce back from negative emotional experiences. Journal of Personality and Social Psychology, 86(4), 320-333. doi: 10.1037/0022-3514.86.2.320

Wang, Y., \& Kong, F. (2014). The Role of Emotional Intelligence in the Impact of Mindfulness on Life Satisfaction and Mental Distress. Social Indicators Research, 116(3), 843-852. doi: 10.1007/s11205-01 3-0327-6

\section{Manolete S. Moscoso}

University of South Florida, Tampa, Florida, USA

Universidad de San Martin de Porres, Lima, Perú

Ph.D. en Psicología, cuenta con investigaciones en estrés crónico, mindfulness, depresión, distrés emocional, ira y hostilidad. Su línea de investigación está centrada en temas relacionados al estrés crónico, emociones y enfermedad (cáncer).

ORCID: 0000-0001-8713-5749

mmoscoso@health.usf.edu 